\title{
A comparison of the influencing factors of chronic pain and quality of life between older Koreans and Korean-Americans with chronic pain: a correlational study
}

\author{
Sun Ju Chang ${ }^{1} \cdot$ Hee Jun $\mathrm{Kim}^{2}$ (D) $\cdot$ Hee-Soon Juon ${ }^{3} \cdot$ Hyunjeong Park $^{4} \cdot$ Seung Woo Choi ${ }^{5}$ Kyung-eun Lee $^{6}$. \\ Hyunju Ryu ${ }^{6}$
}

Accepted: 13 August 2021 / Published online: 30 August 2021

(c) The Author(s) 2021

\begin{abstract}
Background Chronic pain is one of the most common health problems for older adults worldwide and is likely to result in lower quality of life. Living in a different culture may also influence chronic pain and quality of life in older adults. The purpose of this study was to explore how multifaceted elements affect chronic pain and quality of life in older Koreans living in Korea and in older Korean-Americans (KAs) living in the USA.

Methods We conducted a secondary data analysis of data from 270 adults aged 65 years or over (138 Koreans and 132 KAs). We compared the effects of multifaceted elements on pain and quality of life by testing structural equation models (SEMs) for each group, using a maximum likelihood estimation and bootstrapping.

Results SEMs for both Korean and KAs showed that age and depressive symptoms directly affected quality of life. The number of comorbidities and depressive symptoms had mediating effects on quality of life through chronic pain in both groups. In older Koreans only, perceived financial status directly affected quality of life. In older KAs only, sleep quality indirectly affected quality of life through chronic pain.

Conclusion The data showed that multimorbidity and depressive symptoms play critical roles for explaining chronic pain in older Koreans and KAs and ultimately negatively influence quality of life. Future intervention program to improve quality of life in older adults with chronic pain should consider the different cultural aspects affecting quality of life for Koreans and KAs.
\end{abstract}

Keywords Chronic pain · Quality of life $\cdot$ Culture $\cdot$ Structural equation modeling

Hee Jun Kim
hkim20@ajou.ac.kr
Sun Ju Chang
changsj@snu.ac.kr
Hee-Soon Juon
Hee-Soon.Juon@jefferson.edu
Hyunjeong Park
hpark@towson.edu
Seung Woo Choi
schoi51@inha.ac.kr
Kyung-eun Lee
jitta123@ snu.ac.kr
Hyunju Ryu
rhj1110@snu.ac.kr

Hee Jun Kim

changsj@snu.ac.kr

Hee-Soon Juon

Hyunjeong Park

Seung Woo Choi

schoi51@inha.ac.kr

rhj1110@snu.ac.kr 


\section{Introduction}

Persistent, recurrent chronic pain affects $39-70 \%$ of older adults worldwide [1-4] and negatively impacts their daily lives not only by causing discomfort, but also by limiting their activities, contributing to loneliness and social isolation. Chronic pain also influences older adults by costing them time and money to manage their pain and other health care needs $[1,5]$. Older adults with chronic pain often also have chronic diseases, including musculoskeletal disorders (e.g., arthritis), endocrine disorders (e.g., diabetes), and cancers $[6,7]$, that further affect their well-being and complicate their health care needs. Therefore, older adults with chronic pain are likely to experience lower quality of life than those without chronic pain [8].

A biopsychosocial approach to pain is a well-known theoretical framework for understanding multi-dimensional aspects of pain [9]. A range of biological, psychological, and social factors can affect how people perceive pain and aging. For example, age [10], comorbidity [11], depressive symptoms and sleep quality [12], and economic status [13] have been reported as significant factors influencing chronic pain among elderly. In addition, the culture in which people live and other cultural aspects are pivotal to understanding chronic pain in older adults [14, 15]. Immigrant older adults may be particularly vulnerable to chronic pain because they are confronted with the cultural barriers and acculturation [15, 16]. Acculturation is a complex concept and can be defined as a process that occurs when an individual contacts two cultures and experiences substantial change in the culture [17]. Although previous studies have reported an association between acculturation and chronic pain, the results are somewhat sparse by age group; a relatively high level of acculturation was found among young immigrants $[17,18]$, and more acculturated immigrants had a higher prevalence of chronic pain [18]. Among elderly immigrants, a relatively low level of acculturation was reported [19], and acculturation did not directly correlate with somatization [20], which is often associated with pain complaints.

To better understand chronic pain and improve quality of life in older adults with chronic pain, a comprehensive approach that considers multifaceted elements affecting pain and quality of life should precede within a sociocultural context. In this study, we aimed to explore how multifaceted elements, including biological (age, the number of comorbidities), psychological (depressive symptoms, sleep quality), and social (perceived financial status, acculturation) aspects, affect chronic pain and quality of life in older adults with who have chronic pain and the same ethnicity, but who are living in different cultures (Fig. 1). We developed and compared two structural equation models which contained the paths from multifaceted elements to chronic pain and quality of life for older Korean-Americans (KAs) living in the USA and Koreans living in Korea. The hypothetical research framework is illustrated in Fig. 1.
Fig. 1 Research framework of this study

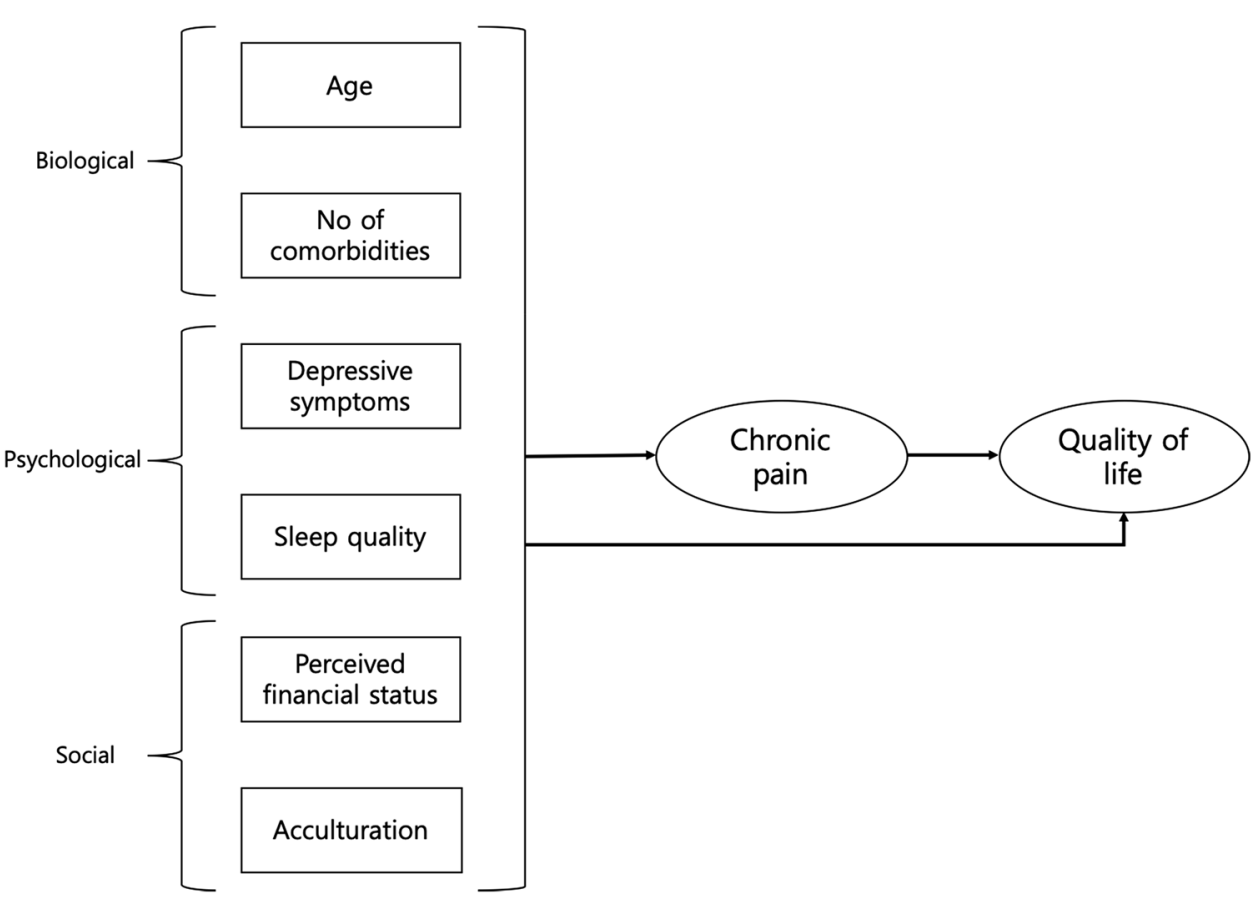




\section{Methods}

\section{Design}

This study was a secondary data analysis of cross-sectional data from a previous study for exploring intra-ethnic differences in chronic pain and associated factors [11]. In order to compare the effects of multifaceted elements on pain and quality of life among older Koreans and KAs with chronic pain, we used a model testing correlation design.

\section{Participants}

A total of 270 older adults (138 for Koreans living in Korea; 132 for KAs living in the USA) participated in the original study. Older adults ( $\geq 65$ years of age) with chronic pain for the last 3 months, no cognitive impairment on the MiniMental Status Examination (score $\geq 24$ ) were included in the study. Older adults with severe medical conditions, such as uncontrolled hypertension and acute pain, daily opioids use, or acute symptoms that interfered with data collection were excluded from the study. Although the participants were recruited in Korea and the USA simultaneously, they were not matched.

The sample size requirements for the structural equation modeling approach have diverse rules-of-thumb methods, such as 5-15 cases per measurements variable [21-23]. Given that we used nine measurement variables in this study, a range of 45-135 cases was needed in each of the two groups studied (Koreans and KAs). Hence, the sample size in this study was considered appropriate for building structural equation models.

\section{Measurements}

\section{Background characteristics}

For this study, we retrieved data from the original study on: age, gender, perceived financial status (very hard to make a living/a little hard to make a living/not hard to make a living at all), the number of comorbidities, and the level of acculturation (for only older KAs) [11]. The level of acculturation was assessed by asking questions about preferred foods, customs, music, language, and close friendship on a 5-point Likert scale [24, 25]. The possible score range was from one to five, with higher scores reflecting greater acculturation.

\section{Depressive symptoms}

The Korean version of the Patient Health Questionnaire-9 was used to assess the level of depressive symptoms in older adults [26]. This self-administered measure, which consists of nine items, asked the participants how often they have experienced nine problems related to depressive symptoms in the last 2 weeks. For each item, participants could choose one of the four responses ranging from "not at all" $(0)$ to "nearly every day" (3). The possible score range was 0-27. The higher the total score, the higher the level of depressive symptoms. The coefficients of Cronbach's alpha were 0.92 in a previous study [26], and 0.86 for Koreans, and 0.80 for KAs in this study, respectively.

\section{Sleep quality}

Sleep quality of the participants was assessed by the Korean version of the Pittsburg Sleep Quality Index [27]. Among 24 items (19 self-administered and five reported by someone sleeping with the participant), this study used the 19 items designed to be self-administered that asked that how often the participant had difficulties sleep in the past month. For each item, the participant could choose one of the four responses ranging from "not during the past month" to "three or more times a week". The global score was yielded by summing the seven components generated by the combination of the items according to the scoring guideline. The possible score range was $0-21$. The higher the global score, the worse the sleep quality. The Cronbach's alpha coefficients were 0.84 in a previous study [27], and 0.72 for Koreans, and 0.79 for KAs in this study, respectively.

\section{Chronic pain}

The Korean version of the Brief Pain Inventory-Short Form was used to assess two domains including the pain severity and pain interference [28]. Four items for the pain severity (now, average, worst, least) and nine for the pain interference (e.g., general activity, mood, walking ability, ordinary work, interpersonal relation, sleep, enjoying life) asked the participant to choose from an 11-point scale ranging from "no pain" or "no interference" $(0)$ to "pain as bad as you can imagine" or "interferes completely" (10). The total scores were calculated by summing all items in each domain in accordance with the scoring guideline [29]. The possible score range was $0-40$ for the severity and $0-90$ for the interference. The higher the score in each domain, the worse the pain or the more pain interferes with daily life, respectively. The study for the development of the Korean version reported the alpha coefficients were 0.85 for the pain severity and 0.93 for the pain interference [28]. In this study, the alpha coefficients were 0.90 for the pain severity and 0.92 for the pain interference in Koreans and 0.86 and 0.91 , respectively, in KAs. 


\section{Quality of life}

The Korean version of the Euro Quality of Life Questionnaire 5-Dimensional Classification-3L (EQ-5D) was used to assess quality of life [30]. This measure consists of five items including mobility, self-care, daily activities, pain/discomfort, and anxiety/depression using a three-level scale as calculating an EQ-5D value and one item for self-rated health status using a visual analog scale (EQ VAS). The possible score range was $0-1$ for the EQ-5D value and $0-100$ for the EQ VAS. The higher the scores, the better the quality of life. The study for the development of the Korean version reported acceptable validity [30]. In this study, the alpha coefficients were 0.81 for Koreans and 0.79 for KAs.

\section{Data collection}

Data collection for the original study was carried out from November 2018 to March 2019 in Maryland, U.S., and Seoul, Korea. The participants were simultaneously recruited from a senior welfare center in Seoul, Korea, and from multiple sites, including Korean senior centers, Korean churches, and elderly day care centers, in Maryland, U.S. The recruitment places were selected to make the functional status of the participants in Korea and in the USA similar. Basically, the participants filled out the questionnaires by themselves; however, the researchers assisted the completion of the questionnaires when the participants want.

\section{Ethical consideration}

The original study was approved by the institutional review boards (IRBs) at the Towson University in the U.S. (\#1,806,036,244) and Seoul National University in Korea (1808/002-009). This secondary data analysis was also approved by the IRB of the Seoul National University (E2005/003-006). When the older adults showed a willingness to participate in the study, the researchers provided sufficient information on the study purpose, time for the survey, possible benefits and risks, and freedom of voluntarily withdrawal from the study without any disadvantage. Among those who understood the purpose of the study and wished to participate in it, the researchers screened the eligibility in accordance with the inclusion and exclusion criteria. Then, written informed consent from all participants was obtained.

\section{Data analysis}

IBM SPSS version 23.0 and AMOS version 23.0 were used to analyze the data. First, all data were reviewed to check for the presence of missing values. Approximately $0.2 \%$ of the data were missing, so they were dealt with expectation-maximization. Background characteristics and all study variables were described by mean and standard deviation for continuous data and frequencies and percentage for categorical data. They were also compared by groups (Koreans vs. KAs) using independent t-tests or chi-square tests. In order to check the assumptions for a structural equation modeling analysis, variables including age, the number of comorbidities, depressive symptoms, sleep quality, perceived financial status, acculturation, pain, and quality of life confirmed no signs of multicollinearities using Pearson correlation analyses, and normality using skewness and kurtosis. Then, the measurement equivalence among two groups, which indicates comparability of measurement in the cross-national study [31,32], was confirmed by multiple group confirmatory factor analysis.

The structural equation model in each group was using a maximum likelihood estimation and bootstrapping. The model fit was evaluated using multiple indices including relative $\chi^{2}$ below 3 , goodness of fit index (GFI) above 0.90 , normed fit index (NFI) above 0.90, comparative fit index (CFI) above 0.90, incremental fit index (IFI) above 0.90, and root mean squared error of approximation below 0.10 in each model $[32,33]$. The parameter estimates in each structural equation models were analyzed using standardized regression weights $(\beta)$, standard errors (S.E.), critical ratio (C.R.), and squared multiple correlations (SMC). In addition, the multifaceted elements, including biological (age, the number of comorbidities), psychological (depressive symptoms, sleep quality), and social (perceived financial status, acculturation) aspects on chronic pain and quality of life, were examined for direct, indirect, and total effects, which were reported by standard regression coefficients $(\beta)$ and $p$-values. Acculturation was input in the model of KAs in this study.

\section{Results}

\section{Background characteristics and descriptive statistics for all variables}

The mean age of the Korean participants was 75.60 years, and the mean age of the of the KA participants was 78.33 years (Table 1). Most of the Korean participants perceived their financial status as a little hard to make a living $(47.8 \%)$ or not hard to make a living (40.6\%), and they had an average of 2.2 comorbidities. Meanwhile, more than a half of KAs perceived their financial status as not hard to make a living $(59.1 \%)$, and they had an average of 2.68 comorbidities and the mean level of acculturation was 1.59.

The mean depressive symptoms score was not significantly different between the two groups ( 4.39 for Koreans and 5.55 for KAs). The mean scores for the other variables were significantly different between the two groups: sleep 
Table 1 Background characteristics and variables among the participants $(n=270)$

\begin{tabular}{|c|c|c|c|}
\hline \multirow[t]{2}{*}{ Characteristics } & Koreans $(N=138)$ & $\begin{array}{l}\text { Korean-Americans } \\
(N=132)\end{array}$ & $P$-value \\
\hline & \multicolumn{3}{|l|}{$M \pm \mathrm{SD}$ or $N(\%)$} \\
\hline \multicolumn{4}{|l|}{ Age, years } \\
\hline $65-74$ & $61(44.2)$ & $37(28.0)$ & \\
\hline $75-84$ & $65(47.1)$ & $71(53.8)$ & \\
\hline \multirow[t]{2}{*}{$\geq 85$} & $12(8.7)$ & $24(18.2)$ & \\
\hline & $75.69 \pm 6.53$ & $78.33 \pm 6.68$ & .001 \\
\hline \multicolumn{4}{|l|}{ Gender } \\
\hline Male & $61(44.2)$ & $24(18.2)$ & $<.001$ \\
\hline Female & $77(55.8)$ & $108(81.8)$ & \\
\hline \multicolumn{4}{|l|}{ Financial status } \\
\hline Very hard to make a living & $16(11.6)$ & $10(7.6)$ & .010 \\
\hline A little hard to make a living & $66(47.8)$ & $44(33.3)$ & \\
\hline Not hard to make a living & $56(40.6)$ & $134(59.1)$ & \\
\hline The number of comorbidities (range $0-9$ ) & $2.20 \pm 1.27$ & $2.68 \pm 1.87$ & .014 \\
\hline Level of acculturation (range $0-5$ ) & - & $1.59 \pm 0.04$ & \\
\hline \multicolumn{4}{|l|}{ Variables } \\
\hline Depressive symptoms (range 0-27) & $4.39 \pm 4.85$ & $5.55 \pm 5.34$ & .064 \\
\hline Sleep quality (range $0-21$ ) & $6.54 \pm 3.50$ & $7.69 \pm 4.64$ & .030 \\
\hline \multicolumn{4}{|l|}{ Chronic pain } \\
\hline Severity (range 0-40) & $12.23 \pm 7.95$ & $15.06 \pm 8.72$ & .006 \\
\hline Interference (range 0-90) & $17.28 \pm 14.60$ & $22.92 \pm 18.13$ & .028 \\
\hline \multicolumn{4}{|l|}{ Quality of life } \\
\hline EQ-5D value (range $0-1$ ) & $0.82 \pm 0.12$ & $0.75 \pm 0.18$ & $<.001$ \\
\hline EQ VAS (range 0-100) & $75.78 \pm 16.89$ & $65.62 \pm 20.37$ & $<.001$ \\
\hline
\end{tabular}

quality (6.54 for Koreans, 7.69 for KAs), chronic pain severity (12.23 for Koreans, 15.06 for KAs), chronic pain interference (17.28 for Koreans, 22.92 for KAs), quality of life (0.82 for Koreans, 0.75 for KAs), and self-rated health status (75.78 for Koreans, 65.62 for KAs) (Table 1).

\section{Structural equation modeling in each group}

The results of the structural equation models in older Koreans and KAs with chronic pain appear in Fig. 2 and Table 2. As illustrated in Fig. 2, the fit indices of each model were satisfied with criterion (relative $\chi^{2}$ of 1.384 , GFI of .972 , NFI of .951, CFI of .985, IFI of .986, and RMSEA of .053 for Koreans; relative $\chi^{2}$ of 2.166 , GFI of .961, NFI of .944, CFI of .967, IFI of .969, and RMSEA of .094 for KAs).

In the model for Koreans (Fig. 2 and Table 2), there were two significant paths to chronic pain: the number of comorbidities $(\beta=.231, p=.003)$ and depressive symptoms $(\beta=.359, p<.001)$. In addition, each path from age $(\beta=-.174, p=.015)$, depressive symptoms $(\beta=-.194$, $p=.027)$, perceived financial status $(\beta=.166, p=.025)$, and chronic pain $(\beta=-.727, p<.001)$ to quality of life in Koreans was significant.
In the model for KAs (Fig. 2 and Table 2), there were four significant paths to chronic pain: age $(\beta=.170, p=.019)$, the number of comorbidities $(\beta=.215, p=.003)$, depressive symptoms $(\beta=.424, p<.001)$, and sleep quality $(\beta=.210$, $p=.009)$. In addition, each path from age $(\beta=-.155$, $p=.021)$, depressive symptoms $(\beta=-.178, p=.041)$, and chronic pain $(\beta=-.769, p<.001)$ to quality of life in KAs was significant.

Standardized direct, indirect, and total effects in each model are summarized in Tables 3 and 4. In the model for Koreans (Table 3 ), the number of comorbidities $(\beta=.231$, $p=.034)$ and depressive symptoms $(\beta=.359, p=.004)$ had direct and total effects on chronic pain. Among the variables that had total effects on quality of life, including age $(\beta=-.222, p=.003)$, the number of comorbidities $(\beta=-.256, p=.006)$, depressive symptoms $(\beta=-.455$, $p=.004)$, and chronic pain $(\beta=-.727, p=.002)$, the number of comorbidities and depressive symptoms indirectly affected quality of life through chronic pain even though they did not directly affect it.

In the model for KAs (Table 4$)$, age $(\beta=.170, p=.011)$, the number of comorbidities $(\beta=.215, p=.014)$, depressive symptoms $(\beta=.424, p=.006)$, and sleep quality $(\beta=.210, p=.041)$ had direct and total effects on chronic 
(a) Older Koreans

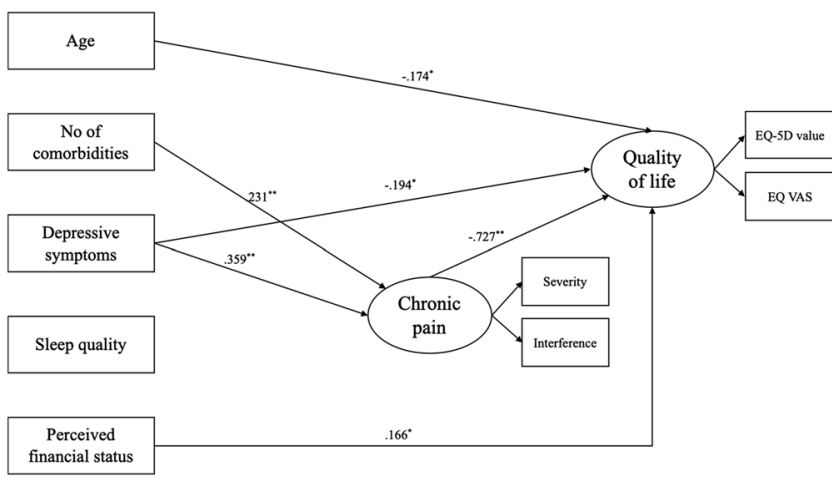

Relative $\chi^{2} 1$ 1.384, GFI .972, NF1 .951, CFI. 985 , IFI .986, RMSEA.053

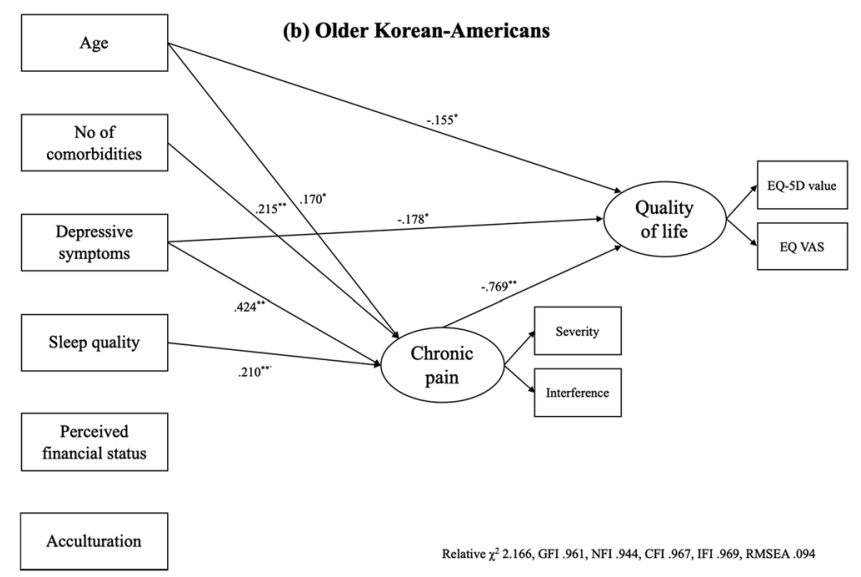

Fig. 2 The results of the structural equation modeling

pain. Among the variables that had total effects on quality of life, including age $(\beta=-.285, p=.011)$, the number of comorbidities $(\beta=-.283, p=.018)$, depressive symptoms $(\beta=-.504, p=.004)$, and chronic pain $(\beta=-.769$, $p=.002)$, the number of comorbidities and depressive symptoms only indirectly influenced quality of life through chronic pain, as in the model for KAs. Although sleep quality had an indirect effect on quality of life through chronic pain, the total effect was not significant.

\section{Discussion}

This study presents potential factors for understanding chronic pain and improving quality of life by exploring relationships among multifaceted elements, chronic pain, and quality of life in older adults with chronic pain. Particularly, this study revealed that there were no statistically significant cultural effects on chronic pain and quality of life by comparing the pathways among older adults who had same ethnicity but living in different cultures. This lack of effect could be explained by the low level of acculturation among the older KAs, in that most of them still maintain a Korean lifestyle despite living in the USA. Several previous studies have also found a lower level of acculturation in older Korean immigrants compared with other Asian immigrants [34, 35]. In fact, additional analysis including acculturation levels for KAs in our structural equation model revealed no significant effects of acculturation on either chronic pain or quality of life (data not shown). These findings suggest that acculturation among elderly immigrants may not be a strong variant predicting health outcomes because they tend to have low level of acculturation in general. Nevertheless, assessing cultural effects on health may be important to better understand the Asian elderly immigrants' health and healthcare use
[19] because low acculturation level may suggest a lower likelihood of adjusting to the new culture.

The findings that the number of comorbidities and depressive symptoms had positive effects on chronic pain in both groups were in line with previous studies. Several studies have reported that the higher the number of comorbidities, the more severe the pain intensity and the longer the pain duration [11, 36-38]. This effect might be because chronic pain often results from other health problems, such as musculoskeletal disorders, which are prevalent in older adults [6, 7]. Regarding depressive symptoms, previous studies have reported that about $13 \%$ of older adults have depression and chronic pain simultaneously $[12,39]$ and that older adults with depression are more vulnerable to chronic pain than those without depression [40, 41]. Although reasons for the coexistence of depression and chronic pain remain unclear, the evidence on claiming neuroinflammation in both diseases has been supported [42]. Multimorbidity and depressive symptoms are believed to play critical roles in chronic pain, especially in older Koreans, given that approximately $73 \%$ of older Koreans have at least two chronic diseases, with osteo/rheumatoid arthritis and low back pain/sciatica among the most prevalent chronic diseases, and that $21 \%$ suffer from depression [1].

The finding that age affected chronic pain in the model for older KAs is concordant with previous studies on chronic pain, which have commonly reported that age is one of the risk factors of chronic pain $[10,42]$ and that chronic pain prevalence tends to increase as age advances [2, 43]. However, this finding was not supported in the model for older Koreans in this study. This discrepancy in the relationship between chronic pain and age in the two groups might be related to the age differences in the older Korean and older KAs in our study. Our Korean sample included a relatively younger group than our KA sample, although the age ranges were similar. Hence, it can be inferred that the relationship 
Table 2 Parameter estimates in each structural equation models $(n=270)$

\begin{tabular}{|c|c|c|c|c|c|c|c|c|c|c|c|}
\hline \multirow[t]{2}{*}{ Exogenous } & \multirow[t]{2}{*}{ Endogenous } & \multicolumn{5}{|c|}{ Koreans $(n=138)$} & \multicolumn{5}{|c|}{ Korean-Americans $(n=132)$} \\
\hline & & $\beta$ & S.E. & C.R. & $p$ & SMC & $\beta$ & S.E. & C.R. & $p$ & SMC \\
\hline Age & Chronic pain & .066 & 0.165 & 0.870 & .384 & .284 & .170 & 0.196 & 2.348 & $.019 *$ & .429 \\
\hline The number of comorbidities & & .231 & 0.859 & 2.993 & $.003 * *$ & & .215 & 0.698 & 2.986 & $.003 * *$ & \\
\hline Depressive symptoms & & .359 & 0.246 & 4.239 & $<.001 * *$ & & .424 & 0.290 & 4.821 & $<.001 * *$ & \\
\hline Sleep quality & & .148 & 0.328 & 1.831 & .067 & & .210 & 0.312 & 2.631 & $.009 *$ & \\
\hline Perceived financial status & & .004 & 1.683 & 0.046 & .963 & & .141 & 2.202 & 1.837 & .066 & \\
\hline Acculturation & & - & - & - & - & & .060 & 0.528 & 0.838 & .402 & \\
\hline Age & Quality of life & -.174 & 0.001 & -2.432 & $.015^{*}$ & .887 & -.155 & 0.001 & -2.308 & $.021 *$ & .955 \\
\hline The number of comorbidities & & -.088 & 0.006 & -1.165 & .244 & & -.118 & 0.005 & -1.746 & .081 & \\
\hline Depressive symptoms & & -.194 & 0.002 & -2.217 & $.027 *$ & & -.178 & 0.002 & -2.041 & $.041 *$ & \\
\hline Sleep quality & & .010 & 0.002 & 0.136 & .892 & & .023 & 0.002 & 0.310 & .756 & \\
\hline Perceived financial status & & .166 & 0.010 & 2.245 & $.025 *$ & & -.039 & 0.018 & -0.521 & .602 & \\
\hline Acculturation & & - & - & - & - & & .050 & 0.004 & 0.775 & .438 & \\
\hline Chronic pain & & -.727 & 0.001 & -6.425 & $<.001 * *$ & & -.769 & 0.001 & -9.509 & $<.001 * *$ & \\
\hline
\end{tabular}

$\beta$ standardized regression weights, S.E. standard errors, C.R. critical ratio, SMC squared multiple correlations

$* p<.05$

$* * p<.01$

Table 3 Direct, indirect, and total effects in the structural equation model for older Koreans with chronic pain $(n=138)$

\begin{tabular}{|c|c|c|c|c|c|c|c|}
\hline \multirow[t]{2}{*}{ Exogenous } & \multirow[t]{2}{*}{ Endogenous } & \multicolumn{2}{|c|}{ Standardized total effect } & \multicolumn{2}{|c|}{ Standardized direct effect } & \multicolumn{2}{|c|}{$\begin{array}{l}\text { Standardized indirect } \\
\text { effect }\end{array}$} \\
\hline & & $\beta$ & $p$-value & $\beta$ & $p$-value & $\beta$ & $p$-value \\
\hline Age & Chronic pain & .143 & .456 & .066 & .456 & & \\
\hline The number of comorbidities & & .231 & $.034 *$ & .231 & $.034 *$ & & \\
\hline Depressive symptoms & & .359 & $.004 * *$ & .359 & $.004 * *$ & & \\
\hline Sleep quality & & .148 & .135 & .148 & .135 & & \\
\hline Perceived financial status & & .004 & .967 & .004 & .967 & & \\
\hline Age & Quality of life & -.222 & $.003 * *$ & -.174 & $.010 * *$ & -.048 & .432 \\
\hline The number of comorbidities & & -.256 & $.006 * *$ & -.088 & .185 & -.168 & $.024 *$ \\
\hline Depressive symptoms & & -.455 & $.004 * *$ & -.194 & .165 & -.261 & $.002 * *$ \\
\hline Sleep quality & & -.097 & .337 & .010 & .994 & -.108 & .104 \\
\hline Perceived financial status & & .163 & .057 & .166 & $.027 *$ & -.003 & .959 \\
\hline Chronic pain & & -.727 & $.002 * *$ & -.727 & $.002 * *$ & & \\
\hline
\end{tabular}

$* p<.05$

$* * p<.01$

between age and chronic pain is more clearly indicated by the inclusion of older adults aged 75 or over who have high pain prevalence and strong pain intensity $[2,44]$.

Both models for older Koreans and KAs with chronic pain showed that age, the number of comorbidities, depressive symptoms, and chronic pain had significant effects on quality of life, and these findings are consistent with previous studies $[8,41,45]$. Among these factors, chronic pain had the strongest effect on quality of life in both groups. As we pointed out earlier, chronic pain in older adults hinders functional independence in everyday activities, limits social interactions, and hampers leisure activities [46, 47]. Health care-related costs to manage chronic pain can also be financially burdensome [1,5]. For those reasons, chronic pain may negatively influence quality of life in older Koreans and KAs in this study.

Perceived financial status had a positive direct effect on quality of life only in the older Koreans, whereas sleep 
Table 4 Direct, indirect, and total effects in the structural equation model for older Korean-Americans with chronic pain $(n=132)$

\begin{tabular}{|c|c|c|c|c|c|c|c|}
\hline \multirow[t]{2}{*}{ Exogenous } & \multirow[t]{2}{*}{ Endogenous } & \multicolumn{2}{|c|}{ Standardized total effect } & \multicolumn{2}{|c|}{ Standardized direct effect } & \multicolumn{2}{|c|}{$\begin{array}{l}\text { Standardized indirect } \\
\text { effect }\end{array}$} \\
\hline & & $\beta$ & $p$-value & $\beta$ & $p$-value & $\beta$ & $p$-value \\
\hline Age & Chronic pain & .170 & $.011^{*}$ & .170 & $.011^{*}$ & & \\
\hline The number of comorbidities & & .215 & $.014 *$ & .215 & $.014^{*}$ & & \\
\hline Depressive symptoms & & .424 & $.006^{* *}$ & .424 & $.006^{* *}$ & & \\
\hline Sleep quality & & .210 & $.041 *$ & .210 & $.041 *$ & & \\
\hline Perceived financial status & & .141 & .098 & .141 & .098 & & \\
\hline Acculturation & & .060 & .346 & .060 & .346 & & \\
\hline Age & Quality of life & -.285 & $.011^{*}$ & -.155 & $.027^{*}$ & -.130 & $.008 * *$ \\
\hline The number of comorbidities & & -.283 & $.018^{*}$ & -.118 & .181 & -.165 & $.011^{*}$ \\
\hline Depressive symptoms & & -.504 & $.004 * *$ & -.178 & .118 & -.326 & $.003 * *$ \\
\hline Sleep quality & & -.138 & .207 & .023 & .785 & -.161 & $.030 *$ \\
\hline Perceived financial status & & -.148 & .095 & -.039 & .609 & -.109 & .098 \\
\hline Acculturation & & .004 & .998 & .050 & .493 & -.046 & .346 \\
\hline Chronic pain & & -.769 & $.002 * *$ & -.769 & $.002 * *$ & & \\
\hline
\end{tabular}

$* p<.05$

$* * p<.01$

quality had a negative indirect effect on quality of life through chronic pain only in the older KAs. A reason for this discrepancy between the two groups may be differences in social security benefits between the two locations that affect financial status. Most of the KAs who participated in this study were receiving Social Security Medicare Program benefits, and they were attending adult daycare centers where they could receive diverse benefits, including meals and health care services. For Korean elders, health insurance is covered by the government, but daily costs for living are relatively higher than in the USA, even in the elderly. The perceived financial status finding agrees with previous studies that reported a significant positive association between financial status and quality of life in older Koreans [48, 49]. That is, the finding on perceived financial status could be supported by the fact that most of the older Koreans in this study experienced more financial difficulties than the older KAs. Regarding sleep quality, the discrepancy between the two groups may be associated with more advanced age of the in KAs in the sample. Aging is associated with sleep quality [50], and poor sleep quality had a significant impact on chronic pain, as well as quality of life, in KAs. Previous studies identified poor sleep quality as leading to chronic pain by increasing pain sensitivity and, in turn, reducing quality of life $[51,52]$. As in the previous studies, greater sleep disturbance in older KAs might negatively affect chronic pain and eventually result in poor quality of life. However, since both perceived financial status in older Koreans and sleep quality in older KAs did not have total effects on quality of life, the findings should be interpreted with a caution.
This study had some potential limitations. First, some variables, such as the number of comorbidities, depressive symptoms, sleep quality, chronic pain, and quality of life, are interrelated so that the associations could be bidirectional $[42,53]$. However, we did not consider the bidirectional relationships because this study aimed to explore multifaceted elements affecting pain and quality of life. Second, as a secondary data analysis, this study used prearranged data. Although major variables affecting chronic pain and quality of life were included in the analyses, this study could not address additional information, such as pain management and immigration status. Lastly, the KAs included in this study were recruited from a region in the USA where a relatively large group of Koreans are living (Korean authentic foods and markets are available, and native language is commonly used), which may have influenced acculturation; thus, generalization of the results is limited.

\section{Conclusion}

This study showed that multifaceted elements, including biological (age, the number of comorbidities), psychological (depressive symptoms, sleep quality), and social (perceived financial status) aspects, affect chronic pain and quality of life in older adults with chronic pain and that there were no cultural effects on chronic pain and quality of life in older adults who had the same ethnicity, but were living in different cultures. Some suggestions can be 
made based on the findings of this study. First, healthcare providers who come across older adults with chronic pain should examine affecting factors and relationships from various standpoints. In particular, when targeting older immigrants, healthcare providers should carefully assess older immigrants' acculturation level using a valid measurement and consider integrating the acculturation level into an intervention program for alleviating chronic pain and improving quality of life. Further studies are needed to examine more comprehensive variables affecting quality of life in older immigrants with chronic pain. In addition, further studies are needed that use multiple measurements, such as a combination of direct (e.g., actigraphy) and indirect (e.g., sleep questionnaire) measurements, to provide a more accurate and extensive view of quality of life.

Acknowledgements This work was supported by Research Resettlement Fund for the new faculty of Seoul National University and Faculty Development and Research Committee Grant (\#15740) from Towson University

Funding This work was supported by Research Resettlement Fund for the new faculty of Seoul National University and Faculty Development and Research Committee Grant (\#15740) from Towson University.

Data availability Due to the nature of this research, participants for this study did not agree for their data to be shared publicly, so supporting data are not available.

\section{Declarations}

Conflict of interest The authors declare no potential conflict of interest with respect to the authorship and/or publication of this article.

Ethical approval The original study was approved by the institutional review boards (IRBs) at the universities in the USA (\#1806036244) and Korea (1808/002-009). This secondary data analysis was also approved by the IRB (E2005/003-006).

Open Access This article is licensed under a Creative Commons Attribution 4.0 International License, which permits use, sharing, adaptation, distribution and reproduction in any medium or format, as long as you give appropriate credit to the original author(s) and the source, provide a link to the Creative Commons licence, and indicate if changes were made. The images or other third party material in this article are included in the article's Creative Commons licence, unless indicated otherwise in a credit line to the material. If material is not included in the article's Creative Commons licence and your intended use is not permitted by statutory regulation or exceeds the permitted use, you will need to obtain permission directly from the copyright holder. To view a copy of this licence, visit http://creativecommons.org/licenses/by/4.0/.

\section{References}

1. Jung, J. H., Park, J. Y., Kim, N. S., \& Park, H. Y. (2016). Status of chronic pain prevalence in the Korean adults. Public Health Weekly Report, KCDC, 8(31), 728-734.
2. Larsson, C., Hansson, E. E., Sundquist, K., \& Jakobsson, U. (2017). Chronic pain in older adults: Prevalence, incidence, and risk factors. Scandinavian Journal of Rheumatology, 46(4), 317-325. https://doi.org/10.1080/03009742.2016.1218543.

3. Bauer, H., Emeny, R. T., Baumert, J., \& Ladwig, K. H. (2016). Resilience moderates the association between chronic pain and depressive symptoms in the elderly. European Journal of Pain (London, England), 20(8), 1253-1265. https://doi.org/10.1002/ ejp.850.

4. Treede, R. D., Rief, W., Barke, A., Aziz, Q., Bennett, M. I., Benoliel, R., et al. (2015). A classification of chronic pain for ICD-11. Pain, 156(6), 1003-1007. https://doi.org/10.1097/j. pain.0000000000000160.

5. Breivik, H., Eisenberg, E., O’Brien, T., \& OPENMinds. (2013). The individual and societal burden of chronic pain in Europe: The case for strategic prioritisation and action to improve knowledge and availability of appropriate care. BMC Public Health, 13, 1229-2458. https://doi.org/10.1186/ 1471-2458-13-1229.

6. Dziechciaz, M., Balicka-Adamik, L., \& Filip, R. (2013). The problem of pain in old age. Annals of Agricultural and Environmental Medicine: AAEM, Spec no. 1, 35-38.

7. Ali, A., Arif, A. W., Bhan, C., Kumar, D., Malik, M. B., Sayyed, Z., et al. (2018). Managing chronic pain in the elderly: An overview of the recent therapeutic advancements. Cureus, 10(9), e3293. https://doi.org/10.7759/cureus.3293.

8. Ferretti, F., Castanha, A. C., Radoan, E. R., Lutinski, J., \& da Silva, M. R. (2018). Quality of life in the elderly with and without chronic pain. Brazilian Journal of Pain, 1(2), 111-115. https://doi.org/10.5935/2595-0118.20180022.

9. Gatchel, R. J., Peng, Y. B., Peters, M. L., Fuchs, P. N., \& Turk, D. C. (2007). The biopsychosocial approach to chronic pain: Scientific advances and future directions. Psychological Bulletin, 133(4), 581-624.

10. Etherton, J., Lawson, M., \& Graham, R. (2014). Individual and gender differences in subjective and objective indices of pain: Gender, fear of pain, pain catastrophizing and cardiovascular reactivity. Applied Psychophysiology and Biofeedback, 39(2), 89-97.

11. Kim, H. J., Chang, S. J., Park, H., Choi, S. W., Juon, H. S., Lee, K. E., \& Ryu, H. (2020). Intra-ethnic differences in chronic pain and the associated factors: An exploratory, comparative design. Journal of Nursing Scholarship: An Official Publication of Sigma Theta Tau International Honor Society of Nursing, 52(4), 389-396. https://doi.org/10.1111/jnu.12564.

12. Ko, K. T., Yu, S. H., Lee, C. Y., Tseng, H. Y., Chiu, Y. F., \& Hsiung, C. A. (2018). Moderating effect of depression on the association between pain and activities of daily living in older adults. Psychogeriatrics, 18(5), 379-387.

13. Feldman, C. H., Dong, Y., Katz, J. N., Donnell-Fink, L. A., \& Losina, E. (2015). Association between socioeconomic status and pain, function and pain catastrophizing at presentation for total knee arthroplasty. BMC Musculoskeletal Disorders, 16, 18. https://doi.org/10.1186/s12891-015-0475-8.

14. Lane, P., \& Smith, D. (2018). Culture, ageing and the construction of pain. Geriatrics (Basel, Switzerland). https://doi.org/10. 3390/geriatrics3030040.

15. Pillay, T., van Zyl, H. A., \& Blackbeard, D. (2014). Chronic pain perception and cultural experience. Procedia-Social and Behavioral Sciences, 113, 151-160. https://doi.org/10.1016/j. sbspro.2014.01.022.

16. Re, T. S., Bragazzi, N. L., Siri, A., Cisneros Puebla, C., Friese, S., Simoes, M., et al. (2017). Effects of acculturation, coping strategies, locus of control, and self-efficacy on chronic pain: Study of chinese immigrant women in italy-Insights from a thematic field analysis. Journal of Pain Research, 10, 13831390. https://doi.org/10.2147/JPR.S115449. 
17. Abraido-Lanza, A. F., Armbrister, A. N., Florez, K. R., \& Aguirre, A. N. (2006). Toward a theory-driven model of acculturation in public health research. American Journal of Public Health, 96(8), 1342-1346. https://doi.org/10.2105/AJPH.2005. 064980.

18. Bui, Q., Doescher, M., Takeuchi, D., \& Taylor, V. (2011). Immigration, acculturation and chronic back and neck problems among Latino-Americans. Journal of Immigrant and Minority Health, 13(2), 194-201. https://doi.org/10.1007/s10903-010-9371-3.

19. Jiang, L., Sun, F., Zhang, W., Wu, B., \& Dong, X. (2019). Health service use among Chinese American older adults: Is there a somatization effect? Journal of the American Geriatrics Society, 67(S3), S584-S589. https://doi.org/10.1111/jgs.15734.

20. Mak, W. W. S., \& Zane, N. W. S. (2004). The phenomenon of somatization among community Chinese Americans. Social Psychiatry and Psychiatric Epidemiology, 39, 967-974.

21. Nunnally, J. C. (1967). Psychometric theory. McGraw-Hill.

22. Stevens, J. (Ed.). (1996). Applied multivariate statistics for the social sciences (3rd ed.). Lawrence Erlbaum Associates.

23. Bentler, P. M., \& Chou, C. (1987). Practical issues in structural modeling. Sociological Methods \& Research, 16, 78-117. https:// doi.org/10.1177/0049124187016001004.

24. Im, E. O., Chee, W., Lim, H. J., Liu, Y., \& Kim, H. K. (2008). Midlife women's attitudes toward physical activity. Journal of Obstetric, Gynecologic, and Neonatal Nursing, 37(2), 203-213. https://doi.org/10.1111/j.1552-6909.2008.00219.x.

25. Im, E. O., Chang, S. J., Ko, Y., Chee, W., Stuifbergen, A., \& Walker, L. (2012). A national internet survey on midlife women's attitudes toward physical activity. Nursing Research, 61(5), 342352. https://doi.org/10.1097/NNR.0b013e31825da85a.

26. Donnelly, P. L., \& Kim, K. S. (2008). The patient health questionnaire (PHQ-9K) to screen for depressive disorders among immigrant Korean American elderly. Journal of Cultural Diversity, 15(1), 24.

27. Sohn, S. I., Kim, D. H., Lee, M. Y., \& Cho, Y. W. (2012). The reliability and validity of the Korean version of the Pittsburgh sleep quality index. Sleep and Breathing, 16(3), 803-812.

28. Yun, Y. H., Mendoza, T. R., Heo, D. S., Yoo, T., Heo, B. Y., Park, H., et al. (2004). Development of a cancer pain assessment tool in Korea: A validation study of a Korean version of the brief pain inventory. Oncology, 66(6), 439-444.

29. Cleeland, C. S. (1991). The Brief Pain Inventory, user guide. Cleeland. Retrieved from https://www.mdanderson.org/content/ dam/mdanderson/documents/Departments-and-Divisions/Sympt om-Research/BPI_UserGuide.pdf.

30. Kim, M., Cho, Y., Uhm, W., Kim, S., \& Bae, S. (2005). Crosscultural adaptation and validation of the Korean version of the EQ-5D in patients with rheumatic diseases. Quality of Life Research, 14(5), 1401-1406.

31. Davidov, E., Meuleman, B., Cieciuch, J., Schmidt, P., \& Billiet, J. (2014). Measurement equivalence in cross-national research. Annual Review of Sociology, 40, 55-75. https://doi.org/10.1146/ annurev-soc-071913-043137.

32. Bae, B. L. (Ed.). (2009). Structural equation modeling with Amos 17.0: Principles and practice (2nd ed.). Cheunglam.

33. Arbuckle, J. L. (2007). Amos TM 16 user's guide. SPSS.

34. Jang, Y., \& Chiriboga, D. A. (2011). Social activity and depressive symptoms in Korean American older adults: The conditioning role of acculturation. Journal of Aging and Health, 23(5), 767-781. https://doi.org/10.1177/0898264310396214.

35. Kim, G., Jang, Y., \& Chiriboga, D. A. (2012). Personal views about aging among Korean American older adults: The role of physical health, social network, and acculturation. Journal of Cross-Cultural Gerontology, 27(2), 139-148. https://doi.org/10. 1007/s10823-012-9165-2.
36. Leong, I. Y., Farrell, M. J., Helme, R. D., \& Gibson, S. J. (2007). The relationship between medical comorbidity and self-rated pain, mood disturbance, and function in older people with chronic pain. The Journals of Gerontology. Series A, Biological Sciences and Medical Sciences, 62(5), 550-555.

37. Scherer, M., Hansen, H., Gensichen, J., Mergenthal, K., RiedelHeller, S., Weyerer, S., et al. (2016). Association between multimorbidity patterns and chronic pain in elderly primary care patients: A cross-sectional observational study. BMC Family Practice, 17, 68. https://doi.org/10.1186/s12875-016-0468-1.

38. Dahan, H., Shir, Y., Velly, A., \& Allison, P. (2015). Specific and number of comorbidities are associated with increased levels of temporomandibular pain intensity and duration. The Journal of Headache and Pain, 16, 528. https://doi.org/10.1186/ s10194-015-0528-2.

39. Mossey, J. M., \& Gallagher, R. M. (2004). The longitudinal occurrence and impact of comorbid chronic pain and chronic depression over two years in continuing care retirement community residents. Pain Medicine (Malden, Mass.), 5(4), 335-348.

40. Ohayon, M. M., \& Stingl, J. C. (2012). Prevalence and comorbidity of chronic pain in the German general population. Journal of Psychiatric Research, 46(4), 444-450. https://doi.org/10.1016/j. jpsychires.2012.01.001.

41. da Silva, M. R., Ferretti, F., da Silva Pinto, S., \& Filho, O. F. T. (2018). Depressive symptoms in the elderly and its relationship with chronic pain, chronic diseases, sleep quality and physical activity level. Brazilian Journal of Pain, 1(4), 293-298. https:// doi.org/10.5935/2595-0118.20180056.

42. Zis, P., Daskalaki, A., Bountouni, I., Sykioti, P., Varrassi, G., \& Paladini, A. (2017). Depression and chronic pain in the elderly: Links and management challenges. Clinical Interventions in Aging, 12, 709-720. https://doi.org/10.2147/CIA.S113576.

43. Docking, R. E., Fleming, J., Brayne, C., Zhao, J., Macfarlane, G. J., Jones, G. T., \& Cambridge City over-75s Cohort Study collaboration. (2011). Epidemiology of back pain in older adults: Prevalence and risk factors for back pain onset. Rheumatology (Oxford, England), 50(9), 1645-1653. https://doi.org/10.1093/ rheumatology/ker175.

44. Cheung, C. W., Choi, S. W., Wong, S. S. C., Lee, Y., \& Irwin, M. G. (2017). Changes in prevalence, outcomes, and help-seeking behavior of chronic pain in an aging population over the last decade. Pain Practice : The Official Journal of World Institute of Pain, 17(5), 643-654. https://doi.org/10.1111/papr.12496.

45. Lemos, B. O., da Cunha, A. R. R., Cesarino, C. B., \& Martins, M. R. I. (2019). The impact of chronic pain on functionality and quality of life of the elderly. Brazilian Journal of Pain, 2(3), 237-241. https://doi.org/10.5935/2595-0118.20190042.

46. Achterberg, W. P. (2019). How can the quality of life of older patients living with chronic pain be improved? Pain Management, 9(5), 431-433. https://doi.org/10.2217/pmt-2019-0023.

47. Santos, F. C., de Moraes, N. S., Pastore, A., \& Cendoroglo, M. S. (2015). Chronic pain in long-lived elderly: Prevalence, characteristics, measurements and correlation with serum vitamin $\mathrm{D}$ level. Revista Dor, 16(3), 171-175. https://doi.org/10.5935/1806-0013. 20150034

48. No, Y. J., Lee, H. K., Lee, W. H., \& Cho, C. L. (2018). Multidimensional life satisfaction and three resources: Economy, health, and relationships. Korean Journal of Public Administration, 56(3), 233-267.

49. Lee, H. J. (2009). An exploratory study on factors affecting quality of life for the elderly: Focusing on socioeconomic and social capital traits. Korean Journal of Stress Research, 17(2), 131-141.

50. Mander, B. A., Winer, J. R., \& Walker, M. P. (2017). Sleep and human aging. Neuron, 94(1), 19-36. https://doi.org/10.1016/j. neuron.2017.02.004. 
51. Bjurstrom, M. F., \& Irwin, M. R. (2016). Polysomnographic characteristics in nonmalignant chronic pain populations: A review of controlled studies. Sleep Medicine Reviews, 26, 74-86. https://doi. org/10.1016/j.smrv.2015.03.004.

52. Veldhuijzen, D. S., Greenspan, J. D., \& Smith, M. T. (2008). Sleep and quality of life in chronic pain. In J. C. Verster, S. R. PandiPerumal, \& D. L. Streiner (Eds.), Sleep and quality of life in medical illness (pp. 187-197). Humana Press.
53. Smith, M. T., \& Haythornthwaite, J. A. (2004). How do sleep disturbance and chronic pain inter-relate? Insights from the longitudinal and cognitive-behavioral clinical trials literature. Sleep Medicine Reviews, 8(2), 119-132. https://doi.org/10.1016/S10870792(03)00044-3.

Publisher's Note Springer Nature remains neutral with regard to jurisdictional claims in published maps and institutional affiliations. 